\title{
Mental Health Concerns of Frontline Workers During the COVID-19 Pandemic: A Scoping Review
}

\author{
Jeavana Sritharan $^{1}$, Thivia Jegathesan ${ }^{2}$, Dharshie Vimaleswaran ${ }^{3} \&$ Ashvinie Sritharan ${ }^{4}$ \\ ${ }^{1}$ Faculty of Health Sciences, Ontario Tech University, Oshawa, Canada \\ ${ }^{2}$ Institute of Medical Sciences, University of Toronto, Toronto, Canada \\ ${ }^{3}$ Faculty of Behavioural Sciences, Yorkville University, Frederiction, Canada \\ ${ }^{4}$ ASKRehab, Ajax, Canada \\ Correspondence: Jeavana Sritharan, Faculty of Health Sciences, Ontario Tech University, Oshawa, Ontario L1G 0C5, \\ Canada. Tel: 289-404-9121. E-mail: jeavana.sritharan@ ontariotechu.ca
}

Received: August 24, 2020 Accepted: September 4, 2020 Online Published: September 14, 2020

doi:10.5539/gjhs.v12n11p89 URL: https://doi.org/10.5539/gjhs.v12n11p89

\begin{abstract}
Objectives: The current COVID-19 pandemic continues to have a significant impact on the mental health of frontline workers worldwide. Currently there are limited published studies addressing mental health issues in frontline workers. The objective of this scoping review is to examine the range of existing global literature on mental health issues reported in frontline workers during the COVID-19 pandemic and to understand what mitigating factors exist.

Methods: The scoping review was guided by the Levac Colquhoun and O'Brien's adapted version of Arkey and O'Malley's framework. We performed a comprehensive search of three databases, Pubmed, APA PsychINFO, and CINAHL, identifying 684 studies. In total, 16 original studies and 4 letters to editors were included in this review.

Results: Of the original studies, 13 were published in China, and the remaining 3 in Italy, Turkey, and Iraq; all letters to editors were published in China. Sources of stress reported in frontline workers across studies included direct contact with COVID-19 patients, isolation, putting loved ones at risk, facing life and death decision making with COVID-19 patients, uncertainty with COVID-19 disease control, limited personal protective equipment, time spent thinking about COVID-19, limited staff/resources/pay, burnout, and stigma. Mental health symptoms and outcomes reported in frontline workers were fear, stress, anxiety, depression, insomnia, burnout, and psychological distress.
\end{abstract}

Conclusion: Findings demonstrate the immediate need to increase mental health awareness and resources at an individual and system wide level. Mental health programs need to be catered towards each unique workplace to provide the necessary resources for frontline workers.

Keywords: mental health, frontline workers, healthcare workers, COVID-19, coronavirus, review

\section{Introduction}

As a result of the novel coronavirus, COVID-19, there continues to be concern over the unparalleled mental health issues that will arise across frontline workers around the world (Sritharan \& Sritharan, 2020). The impact from COVID-19 is incomparable to past outbreaks, as this pandemic has taken a toll on the global population with heightened uncertainty and stress (de Girolamo et al., 2020). Although the global number of individuals infected is still increasing, it is expected that the mental health effects will impact more people and last longer than the virus itself (Kang et al., 2020).

There is an immediate need to protect the welfare of the healthcare workforce who are battling the pandemic at the frontlines. It is imperative to protect the mental wellbeing of healthcare workers who are primarily involved in caring for COVID-19 patients, to ensure the longevity of these workers and a healthy workplace (Blake, Bermingham, Johnson, \& Tabner, 2020). Healthcare workers are not only at a higher risk of COVID-19 infection than others, but they also face psychological distress related to multiple factors that include isolation from loved ones to avoid potential spread of the virus, increased workload and work hours leading to burnout, shortage of protective resources in the workplace, emotional and physical exhaustion, and increased life and death decision making (Blake et al., 2020; Bansal et al., 2020; Huang \& Zhao, 2020; Kang et al., 2020; Zhang et al., 2020a; Lu, Wang, Lin, \& Li, 2020; Wang et 
al., 2020; Wu, Styra, \& Gold, 2020; Gold et al., 2020). Global data on the extent to which healthcare workers are affected mentally by COVID-19 have not been published yet, but it is clear that many of these workers around the world are tremendously impacted by the pandemic (Neto et al., 2020).

Currently, published studies on mental health and frontline workers are primarily originating from Asia and Europe, where the pandemic spread first, followed by very limited studies published in North America. It is anticipated that many studies are in progress to understand the mental health impact, which will only become more apparent in the near future and as peer-reviewed published studies emerge. All published studies are important to review to have an appropriate understanding of emerging mental health issues that will have a global impact.

We conducted a scoping review to examine existing published literature on mental health issues reported in frontline workers during the COVID-19 pandemic. This will provide better understanding on the mental health impact while informing mental health strategies to support frontline workers during and after the pandemic. This review will provide a comprehensive understanding of what current published studies have identified as priority areas of mental health over the course of the pandemic.

\section{Methods}

\subsection{Framework}

A scoping review of peer reviewed literature was conducted using Levac Colquhoun and O'Brien's adapted version of Arkey and O'Malley's framework (Levac, Colquhoun, \& O'Brien, 2010) to identify articles reporting the mental health impact of COVID-19 on frontline workers during the present pandemic. This framework includes six components to guide the review which are 1) identifying research question, 2) identify relevant studies, 3) study selection, 4) charting the data, 5) collating, summarizing and reporting results and 6) consultation (Levac et al., 2010). The scoping review was reported according to Preferred Reporting Items for Systematic reviews and Meta-Analyses extension for Scoping Reviews (PRISMA-ScR).

\subsection{Frontline Worker Definition}

Frontline workers are those employed in services and functions that are deemed essential in preserving life, health, and basic functioning of society during the COVID-19 pandemic (Government of Canada, 2020). Essential work includes a broad spectrum of occupations, but primarily includes healthcare workers, first responders (ex. police, firefighting), critical infrastructure workers (ex. electricity, transportation), social workers (ex. social services), and food/medicine supplying workers (Government of Canada, 2020). For the purpose of this paper, any occupation that falls under the mentioned list above, and are considered 'essential' or 'frontline' in any published available journals are included.

\subsection{Identification of the Research Question}

We developed a broad research question for this scoping review asking what does the published available literature tell us about mental health issues among frontline workers during the COVID-19 pandemic? The emergence of mental health issues is imperative for frontline workers who are directly involved with COVID-19 patients, but also for other essential workers who may be exposed to COVID-19. Specific questions in our scoping review are:

A) What types of mental health concerns are commonly identified among frontline workers during the pandemic?

B) What mitigating factors or methods exist to help improve the mental wellbeing of frontline workers?

\subsection{Eligibility, Information Sources, and Search}

Studies published in English, in peer-reviewed journals, providing discussion or data on any frontline workers during the COVID-19 pandemic, and mental health issues experienced by frontline workers were included in the review. The search strategy included a broad selection of study designs and papers including both quantitative and qualitative studies, letters to editors, commentaries, and support papers to collect all available data given that this is a novel topic with limited original studies. Three electronic databases (Pubmed, APA PsychINFO, and CINAHL) were accessed from May 4th to May 30th, 2020 to identify studies meeting the inclusion criteria. Date of publication was restricted from January 1, 2020 to December 31, 2020 to capture the time frame of the pandemic. The databases were searched using a combination of free-text terms, provided in Supplementary Table 1, Appendix. Free-text terms were expanded and combined using boolean operators using MeSH terms and keywords. We also reviewed reference lists of included papers to ensure we did not miss any relevant original studies or literature.

\subsection{Selection of Studies}

Initial screening of relevant including abstracts titles were reviewed by JS and TJ independently and included in full-text review if they met all of the following criteria; 1 ) original mental health discussion of frontline workers during 
COVID-19, 2) published in English and 3) published in peer-reviewed journals. Articles were excluded from the scoping review if they were duplicate articles already included, review articles, did not discuss the mental health impact of the present pandemic on frontline workers, lacked original data, or had no full text access. Any disagreements between two independent reviewers were resolved through consensus.

\subsection{Data Charting Process and Data Items}

A data charting form was created in Microsoft Excel to report the first author, year of publication, location of study, type of study, occupation of frontline workers, sample size, and mental health variables (symptoms, sources of stress, risk factors and outcomes). The data charting form was reviewed and revised as the search strategy and inclusion criteria was refined. Specific evidence in key content areas around mental health experience and outcomes among frontline workers during COVID-19 was extracted and summarized. Strengths and gaps in the current literature related to the research question were presented, followed by recommendations for future research and policies related to the protection and care of frontline workers during this pandemic.

\subsection{Summarizing and Reporting of Results}

Quantitative studies including prospective, and cross-sectional studies discussing mental health impact among frontline workers during COVID-19 were categorized into the following areas; 1) risk factors (demographic and occupational) and sources of stress, 2) symptoms and mental health outcomes, and 3) mental health service access and interventions (if applicable). Commentaries, letters to editors and support papers reporting prospective or retrospective data directly related to the experience of frontline workers during COVID-19 and the impact on their mental health were analyzed using thematic analysis to identify the deeper experience of frontline workers during this pandemic and to inform mental health policies and interventions for frontline workers during COVID-19.

\section{Results}

\subsection{Selection of Sources of Evidence}

A total of 684 articles were identified across all electronic databases using the search strategy summarized in Supplementary Table 1, Appendix. Two independent reviewers (JS and TJ) screened titles and abstracts of all studies independently resulting in the exclusion of duplicate studies and those that did not meet the inclusion criteria $(\mathrm{n}=634$ studies). As a result, 50 studies were assessed as full-text for eligibility by JS and TJ. Only papers that focused on the direct mental health impact of COVID-19 on frontline workers were included in the scoping review to ensure an accurate picture of the mental health issues surrounding frontline workers during COVID-19. As such 20 articles were retained which included 16 original articles and 4 other types of articles (commentaries, letters to editors and support papers) (Figure 1).

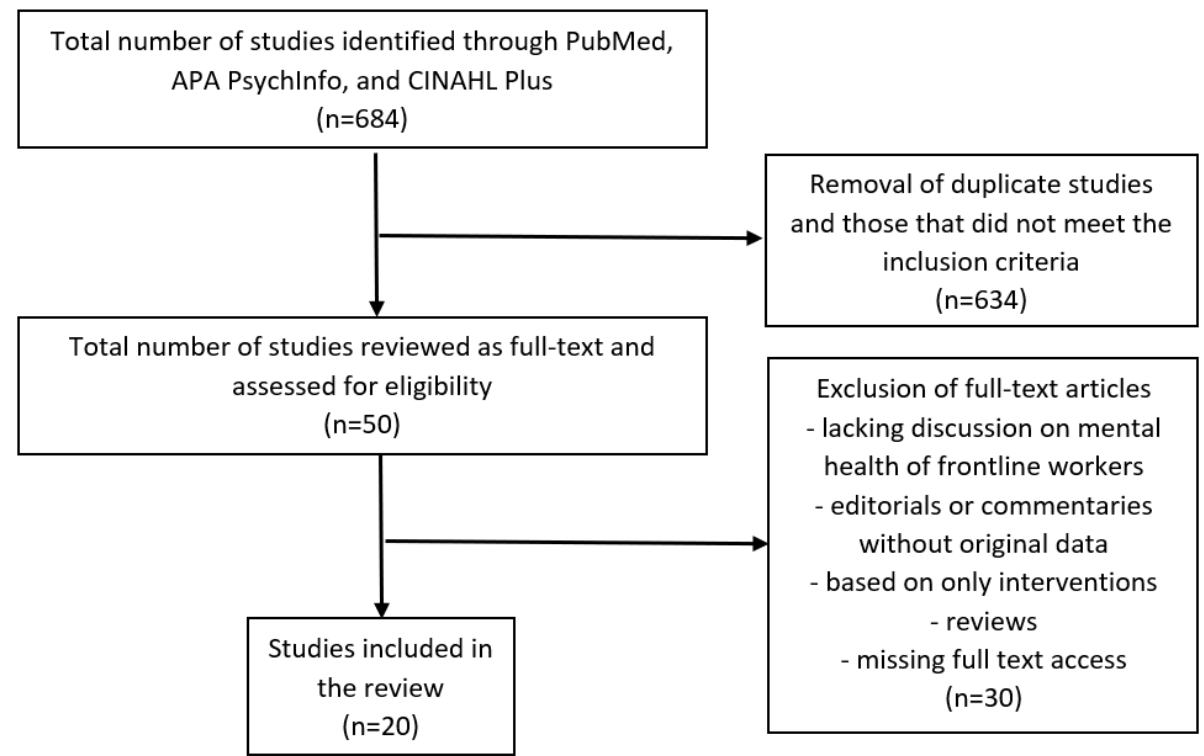

Figure 1. Flow diagram presenting the breakdown of the number of studies identified, reviewed, and included in the scoping review, resulting in the inclusion of 20 studies 


\subsection{Characteristics of Included Studies}

Study design details, participants and mental health outcomes of interest for 16 included original articles are reported in Table 1. General study details of included letters to editors are also summarized in Table 1. Among the 16 original articles, almost all the studies were prospective cross-sectional surveys (93.7\%) using online questionnaires, and one study (6.3\%) was an interview study. Geographically, most of the studies were conducted in China (81.3\%) and remaining 3 studies were done in Italy, Iraq and Turkey. The study sample size ranged from 20 to 7236 frontline workers all of which were healthcare workers in a hospital or healthcare facility. A total of 12 studies assessed the mental health impact across multiple broad healthcare workers which included doctors, nurses, administrative or other medical staff while two studies reported only on nurses and two on only doctors (Table 1). Among the four other types of articles, all articles were letters to the editor reporting a short prospective quantitative study on mental health of healthcare workers during COVID-19 in China. Letters to the editors discussed healthcare workers in general, and surgical staff. 
Table 1. Descriptions of the 16 included original studies and 4 letters to editors in the scoping review, all published in 2020.

\begin{tabular}{|c|c|c|c|c|c|c|}
\hline \multicolumn{7}{|c|}{ Original Articles } \\
\hline $\begin{array}{l}\text { First Author, } \\
\text { Year }\end{array}$ & $\begin{array}{l}\text { Location of } \\
\text { Study }\end{array}$ & Occupation & $\begin{array}{l}\text { Sample } \\
\text { Size }\end{array}$ & Study Design & $\begin{array}{l}\text { Method of Data } \\
\text { Collection }\end{array}$ & $\begin{array}{l}\text { Mental Health Risk Factors and Outcomes } \\
\text { Collected }\end{array}$ \\
\hline Abdulah, 2020 & Iraq & Doctors & 268 & Cross-sectional & $\begin{array}{l}\text { Online } \\
\text { questionnaire }\end{array}$ & Demographic information, stress, insomnia \\
\hline Barello, 2020 & Italy & $\begin{array}{l}\text { Doctors, nurses, other } \\
\text { health professionals }\end{array}$ & 1,153 & Cross-sectional & $\begin{array}{l}\text { Online } \\
\text { questionnaire }\end{array}$ & $\begin{array}{l}\text { Demographic information, psychological stress, sleep } \\
\text { quality, physical health, emotional exhaustion }\end{array}$ \\
\hline Elbay, 2020 & Turkey & Doctors & 442 & Cross-sectional & $\begin{array}{l}\text { Online } \\
\text { questionnaire }\end{array}$ & Demographic information, anxiety, depression, stress \\
\hline Huang, 2020 & China & $\begin{array}{l}\text { Doctors, } \\
\text { administrative staff }\end{array}$ & 7,236 & Cross-sectional & $\begin{array}{l}\text { Online } \\
\text { questionnaire }\end{array}$ & $\begin{array}{l}\text { Demographic information, depression, anxiety, sleep } \\
\text { quality }\end{array}$ \\
\hline Kang, 2020 & China & Doctors, nurses & 994 & Cross-sectional & $\begin{array}{l}\text { Online } \\
\text { questionnaire }\end{array}$ & $\begin{array}{l}\text { Demographic information, mental health assessment, } \\
\text { exposure to COVID-19, mental healthcare services } \\
\text { accessed, psychological needs }\end{array}$ \\
\hline Lai, 2020 & China & Doctors, nurses & 1,257 & Cross-sectional & $\begin{array}{l}\text { Online } \\
\text { questionnaire }\end{array}$ & $\begin{array}{l}\text { Demographic information, exposure to COVID-19, } \\
\text { depression, anxiety, insomnia, psychological distress }\end{array}$ \\
\hline Liu, 2020 & China & Healthcare workers & 512 & Cross-sectional & $\begin{array}{l}\text { Online } \\
\text { questionnaire }\end{array}$ & Demographic information, anxiety \\
\hline $\mathrm{Lu}, 2020$ & China & $\begin{array}{l}\text { Doctors, } \\
\text { administrative staff }\end{array}$ & 2,299 & Cross-sectional & $\begin{array}{l}\text { Online } \\
\text { questionnaire }\end{array}$ & $\begin{array}{l}\text { Demographic information, exposure to COVID-19, } \\
\text { fear, depression, anxiety }\end{array}$ \\
\hline Mo, 2020 & China & Nurses & 210 & Cross-sectional & $\begin{array}{l}\text { Online } \\
\text { questionnaire }\end{array}$ & Demographic information, stress, anxiety \\
\hline Qi, 2020 & China & Healthcare workers & 801 & Cross-sectional & $\begin{array}{l}\text { Online } \\
\text { questionnaire }\end{array}$ & Demographic information, anxiety, insomnia \\
\hline Sun, 2020 & China & Nurses & 20 & $\begin{array}{l}\text { Phenomenological } \\
\text { approach (qualitative) }\end{array}$ & $\begin{array}{l}\text { Interviews (face to } \\
\text { face, telephone) }\end{array}$ & Psychological experience \\
\hline Wang, 2020 & China & Doctors, nurses & 123 & Cross-sectional & $\begin{array}{l}\text { Online } \\
\text { questionnaire }\end{array}$ & $\begin{array}{l}\text { Demographic information, sleep, psychological } \\
\text { distress }\end{array}$ \\
\hline $\mathrm{Wu}, 2020$ & China & Medical staff & 2,210 & Cross-sectional & $\begin{array}{l}\text { Online } \\
\text { questionnaire }\end{array}$ & Psychological status \\
\hline Xiao, 2020 & China & Healthcare workers & 958 & Cross-sectional & $\begin{array}{l}\text { Online } \\
\text { questionnaire }\end{array}$ & Demographic information, anxiety, depression \\
\hline
\end{tabular}




\begin{tabular}{|c|c|c|c|c|c|c|}
\hline Zhang, 2020a & China & $\begin{array}{l}\text { Doctors, nurses, } \\
\text { administrative staff, other } \\
\text { medical staff }\end{array}$ & 1,563 & Cross-sectional & $\begin{array}{l}\text { Online } \\
\text { questionnaire }\end{array}$ & $\begin{array}{l}\text { Demographic information, COVID19 exposure, } \\
\text { insomnia, anxiety, depression }\end{array}$ \\
\hline Zhou, 2020 & China & Doctors, nurses & 1,001 & Cross-sectional & $\begin{array}{l}\text { Online } \\
\text { questionnaire }\end{array}$ & Depression, anxiety, insomnia, psychological distress \\
\hline \multicolumn{7}{|c|}{ Letter to Editor Articles } \\
\hline $\begin{array}{l}\text { First Author, } \\
\text { Year }\end{array}$ & $\begin{array}{l}\text { Location of } \\
\text { Study }\end{array}$ & Occupation & $\begin{array}{l}\text { Sample } \\
\text { Size }\end{array}$ & Study Design & $\begin{array}{l}\text { Method of Data } \\
\text { Collection }\end{array}$ & $\begin{array}{l}\text { Mental Health Risk Factors and Outcomes } \\
\text { Collected }\end{array}$ \\
\hline $\mathrm{Du}, 2020$ & China & Healthcare workers & 134 & Cross-sectional & $\begin{array}{l}\text { Online } \\
\text { questionnaire }\end{array}$ & $\begin{array}{l}\text { Demographic information, COVID-19 exposure, } \\
\text { access to medical and psychological supplies, } \\
\text { self-efficacy, sleep, stress, depression, anxiety }\end{array}$ \\
\hline $\mathrm{Li}, 2020$ & China & Medical staff & 948 & Cross-sectional & $\begin{array}{l}\text { Online } \\
\text { questionnaire }\end{array}$ & $\begin{array}{l}\text { Demographic information, insomnia, psychological } \\
\text { status }\end{array}$ \\
\hline $\mathrm{Xu}, 2020$ & China & Surgical staff & 120 & Prospective cohort & $\begin{array}{l}\text { Anxiety and } \\
\text { depression scales }\end{array}$ & Demographic information, anxiety, depression \\
\hline Zhang, 2020b & China & Healthcare workers & 304 & Mixed methods & $\begin{array}{l}\text { Interviews, } \\
\text { questionnaire }\end{array}$ & $\begin{array}{l}\text { Demographic information, access to PPE, exposure to } \\
\text { COVID-19, Physical health, anxiety, depression, } \\
\text { psychological distress, job satisfaction }\end{array}$ \\
\hline
\end{tabular}




\subsection{Summary of Findings}

Included studies were categorized into 3 categories; 1) risk factors and sources of stress, 2) symptoms and mental health outcomes, and 3) mental health service access and interventions. All 20 studies discuss at least one source of stress or risk factor reported by frontline workers along with mental health symptoms and outcomes during the COVID-19 pandemic. Only one study discusses mental health services accessed.

In this review, few studies reported demographic risk factors associated with increased risk of mental health issues in frontline healthcare workers. One study reported lower education being associated with increased insomnia and another reporting increased insomnia among females than males (Zhang et al., 2020a; Abdulah \& Musa, 2020). Females also reported higher depression, anxiety, and emotional exhaustion than males across two studies (Xiao et al., 2020b; Barello, Palamenghi, \& Graffigna, 2020). Zhang et al. (2020a) explored insomnia symptoms among medical staff identifying multiple risk factors such as low educational level, isolation, worry and fear of being infected with COVID-19, lack of psychological support from media or news outlets regarding the outbreak, and uncertainty surrounding appropriate COVID-19 disease control (Zhang et al., 2020a). With respect to occupation two studies reported nurses at higher risk of experiencing mental health issues than physicians (Zhang et al., 2020a; Barello et al., 2020). Exposure to COVID-19 patients was a major source of stress reported among 14 studies followed by being in isolation and quarantine from loved ones in 6 studies. Key sources of stress reported by frontline workers during the COVID-19 pandemic reported in included studies are shown in Table 2.

Table 2. Sources of stress related to frontline workers during the COVID-19 pandemic reported in the 20 included studies in the scoping review

\begin{tabular}{|c|c|}
\hline Sources of Stress & Original Studies (OS) and Editorials (E) \\
\hline \multirow[t]{2}{*}{$\begin{array}{l}\text { Direct contact with COVID-19 infected patients } \\
\text { (confirmed/symptomatic and asymptomatic cases) }\end{array}$} & $\begin{array}{l}\text { OS: Abdulah et al., 2020; Barello et al., 2020; Elbay et al., 2020; } \\
\text { Huang et al., 2020; Kang et al., 2020; Lai et al., 2020; Lu et al., } \\
\text { 2020; Sun et al., 2020; Wang et al., 2020; Wu W et al., 2020, Liu } \\
\text { et al. 2020, Xiao et al., 2020b }\end{array}$ \\
\hline & E: Du et al., 2020; Li et al., 2020; Xu et al. \\
\hline \multirow[t]{2}{*}{ Isolation or quarantine especially from loved ones } & $\begin{array}{l}\text { OS: Huang et al., 2020; Kang et al., 2020; Lai et al., 2020; Lu et } \\
\text { al., 2020; Zhang et al., 2020a }\end{array}$ \\
\hline & E: Li et al., 2020 \\
\hline \multirow{2}{*}{$\begin{array}{l}\text { Putting loved ones at risk of infection or knowledge of loved ones } \\
\text { being infected }\end{array}$} & OS: Kang et al., 2020; Lu et al., 2020; Sun et al., 2020 \\
\hline & E: Du et al., 2020 \\
\hline
\end{tabular}

Facing life and death decision making around critical COVID-19 patients

OS: Lu et al., 2020; Wu W et al., 2020

Uncertainty around COVID-19 disease control in the OS: Sun et al., 2020; Zhang et al., 2020a
workplace/community

OS: Lu et al., 2020; Mo et al., 2020, Xiao et al. 2020b

Shortage or limited personal protective equipment

E: Zhang et al., 2020b

Time spend focusing or thinking about COVID-19 due to occupation

OS: Huang et al., 2020

OS: Elbay et al., 2020; Mo et al., 2020; Sun et al., 2020,

Increased burnout/stress, staff or resource shortages/low pay

E: Du et al., 2020; Li et al., 2020

Stigma related social rejection and prejudice due to high risk occupation

OS: Lu et al., 2020

Depression, anxiety, and sleep quality/insomnia were the three most common reported mental health symptoms experienced by frontline healthcare workers, followed by stress and fear. Three studies reported overall scores of anxiety and depression but did not report overall rates of these symptoms among frontline workers (Kang et al., 2020, Wu et al., 2020, Xu et al., 2020). A cross-sectional study identified that one in four healthcare workers reported sleep issues more than those in other occupations (Huang \& Zhao, 2020). This finding was attributed to 
the increased work intensity and time, resulting in increased chronic stress and psychological distress (Huang \& Zhao, 2020). Sleep disturbance was also prevalent in healthcare workers in other included cross-sectional studies (Wang et al., 2020; Wu et al., 2020), associated with increased depression and exposure to COVID-19 patients (Wang et al., 2020). The percent of negative mental health symptoms and outcomes experienced by frontline workers during the COVID-19 pandemic across included studies is shown in Table 3. Mental health services and resources accessed by frontline workers reported in one study by Kang et al. (2020) (Table 1) included non-specific psychological materials and resources and counselling or psychotherapy. No other details of mental health service access and resources were discussed in any of the remaining 19 articles.

One qualitative study on nurses in China was included, which reported nurses experienced increased negative emotions in the early stage of the pandemic, as the workload increased with the number of infected patients increasing (Sun et al., 2020). This was followed by the nurses using coping mechanisms such as distraction, humour, isolation to actively attempt to self-care (Sun et al., 2020). They also experienced support from loved ones, colleagues, and members of the society which helped to mediate stress and increase positive emotions. The nurses felt positive emotions alongside the negative emotions, improving their confidence, especially with increased training and practice as the pandemic continued (Sun et al., 2020). Another cross-sectional study in China identified that mental health, psychological distress, and sleep quality substantially improved in healthcare workers in the late stage of the outbreak (Zhou et al., 2020). The study attributed the improvement to effective psychosocial measures that were implemented.

Table 3. Negative mental health symptoms and outcomes among frontline workers during the COVID-19 pandemic reported in included studies in the scoping review

\begin{tabular}{|c|c|c|c|}
\hline $\begin{array}{c}\text { Mental Health Symptoms and } \\
\text { Outcomes }\end{array}$ & $\begin{array}{c}\text { Study } \\
\text { (First Author, Year) }\end{array}$ & Occupation & $\begin{array}{c}\text { Percentage of Participants } \\
\text { Reporting Symptoms and } \\
\text { Outcomes }(\%)\end{array}$ \\
\hline \multirow[t]{3}{*}{ Fear } & Lu et al, 2020 & Medical Staff & 70.6 \\
\hline & Sun et al, 2020 & Administrative Staff & 58.4 \\
\hline & & Nurses & 100.0 \\
\hline \multirow[t]{6}{*}{ Stress } & Abdulah et al, 2020 & Doctors & 93.7 \\
\hline & Du et al, 2020 & Healthcare workers & 59.0 \\
\hline & Elbay et al, 2020 & Healthcare workers & 41.2 \\
\hline & Mo et al, 2020 & Nurses & 100.0 \\
\hline & Zhang et al, 2020 & Medical Staff & 73.4 \\
\hline & Zhou et al, 2020 & Healthcare workers & 27.5 \\
\hline \multirow[t]{12}{*}{ Anxiety } & Du et al, 2020 & Healthcare workers & 20.1 \\
\hline & Elbay et al, 2020 & Healthcare workers & 51.6 \\
\hline & Huang et al, 2020 & Healthcare workers & 35.6 \\
\hline & Lai et al, 2020 & Healthcare workers & 44.6 \\
\hline & Liu et al, 2020 & Healthcare workers & 12.5 \\
\hline & Lu et al, 2020 & Medical Staff & 25.5 \\
\hline & & Administrative staff & 18.7 \\
\hline & Sun et al, 2020 & Nurses & 75.0 \\
\hline & Wang et al, 2020 & Healthcare workers & 18.3 \\
\hline & Xiao et al, 2020 & Healthcare workers & 54.1 \\
\hline & Zhang et al, 2020 & Medical staff & 44.7 \\
\hline & Zhang SX et al, 2020 & Healthcare workers & 28.0 \\
\hline
\end{tabular}




\begin{tabular}{|c|c|c|c|}
\hline \multirow[t]{10}{*}{ Depression } & Du et al, 2020 & Healthcare workers & 12.7 \\
\hline & Elbay et al, 2020 & Healthcare workers & 64.7 \\
\hline & Huang et al, 2020 & Healthcare workers & 19.8 \\
\hline & Lai et al, 2020 & Healthcare workers & 50.4 \\
\hline & Lu et al, 2020 & Medical staff & 12.1 \\
\hline & & Administrative staff & 8.2 \\
\hline & Wang et al, 2020 & Healthcare workers & 57.9 \\
\hline & Xiao et al, 2020 & Healthcare workers & 57.3 \\
\hline & Zhang et al, 2020 & Medical staff & 50.7 \\
\hline & Zhang SX et al, 2020 & Healthcare workers & 30.6 \\
\hline \multirow[t]{9}{*}{ Insomnia/Decreased Sleep } & Abdulah et al, 2020 & Doctors & 68.3 \\
\hline & Barello et al, 2020 & Healthcare workers & 55.3 \\
\hline & Elbay et al, 2020 & Healthcare workers & 78.4 \\
\hline & Huang et al, 2020 & Healthcare workers & 23.6 \\
\hline & Lai et al, 2020 & Healthcare workers & 34.0 \\
\hline & Li et al, 2020 & Medical Staff & 32.8 \\
\hline & Wang et al, 2020 & Healthcare workers & 38.0 \\
\hline & Zhang et al, 2020 & Medical staff & 36.1 \\
\hline & Zhou et al, 2020 & Healthcare workers & 16.8 \\
\hline \multirow[t]{2}{*}{ Psychological Distress } & Lai et al, 2020 & Healthcare workers & 71.5 \\
\hline & Zhang SX et al, 2020 & Healthcare workers & 20.1 \\
\hline
\end{tabular}

*Wu, 2020; Kang, 2020; Xu, 2020 did not report percentage of healthcare worker participants reporting these specific mental health symptoms and outcomes

\section{Discussion}

This is one of the very few reviews addressing mental health issues among frontline workers during the COVID-19 pandemic. All included studies reported on healthcare workers, specifically doctors, nurses, and medical/hospital staff. It is important to mention that there are other health related frontline workers such as personal support workers, auxiliary staff (ex. custodians, housekeeping), mental healthcare workers, respiratory therapists, and paramedics. Although findings are primarily from China, a better understanding of healthcare workers' mental health during the pandemic is important as the number of cases continue to increase in Europe and North America. This review of current literature could drive and inform research and policies during these unprecedented times.

Among mental health symptoms of depression and anxiety, sleep disturbances and insomnia were commonly reported among healthcare workers (Huang \& Zhao, 2020; Wang et al., 2020; Wu et al., 2020). Sleep issues were also exacerbated by exposure to COVID-19 and increased reports of depression and anxiety suggesting a cumulative effect of mental health issues (Wang et al., 2020). Having a low educational level or being female was also associated with increased insomnia (Zhang et al., 2020a; Abdulah \& Musa, 2020; Barello et al., 2020). Socioeconomic stressors, specifically lower education or income levels, are linked to adverse mental health outcomes (Gold et al., 2020). Sleep quality is also influenced by self-efficacy, which provides a positive mental state helping medical staff cope in their high-intensity work (Xiao et al., 2020a). For instance, it is common practice for nurses to work in shifts, however during the pandemic many healthcare workers, including nurses, were and are continuing to work overtime or excessive hours (Zhang et al., 2020a; Xiao et al., 2020a). The increased workload and work hours, essentially burnout, impacts the sleep patterns of workers, which can lead to adverse long-term effects on their sleep patterns. Increased disturbance on sleep patterns can lead to prolonged insomnia, disrupting the circadian rhythm and ultimately having long term effects on the health of these workers (Zhang et al., 2020a; Xiao et al., 2020a). 
Healthcare worker wellness is also significantly linked to stress and burnout in the workplace (Barello et al., 2020). This can lead to increased errors or malpractice, and may lead to increased alcohol and drug use, suicide, and divorce (Bansal et al., 2020; Gold et al., 2020). This can also be compounded by pre-existing mental health disorders (Gold et al., 2020). Another contributing factor are the changing personal protective equipment recommendations and unclear standards directed at healthcare workers over the course of this pandemic (Zhang et al., 2020b; Bansal et al., 2020). This can cause increased depression, stress, and anxiety based on uncertainty and confusion during the outbreak (Zhang et al., 2020b).

Stigma is another major aspect affecting the mental health of healthcare workers. Stigma is related to social rejection, prejudice, and the fear that workers may be infected because of their close contact with infected patients (Park, Lee, Park, \& Choi, 2018; Lu et al., 2020). This can lead to increased stress and eventually a decline in mental health. Looking at the past SARS and MERS outbreaks, there is evidence that hospital staff who worked directly with these patients demonstrated an increased risk of post-traumatic stress disorder (PTSD) after the outbreaks had passed (Min Lee, Kang, Cho, Kim, \& Park, 2018).

All healthcare workers need to be protected and supported during this time, especially nursing staff, to avoid a mental health spillover causing a secondary pandemic of mental distress (Choi, Heilemann, Fauer, \& Mead, 2020). Aside from primarily treating COVID-19 patients, these workers are also at risk of increased mental health issues stemming from the shortage of personal protective equipment, patients not knowing or not being forthcoming about their COVID-19 diagnosis, fear of bringing the virus home to loved ones, and the struggles with facing critical patients where life and death decisions need to be made (Lu et al., 2020; Mo et al., 2020; Gold et al., 2020). These stressful situations are internalized by healthcare workers leading to increased anxiety and depression symptoms. In Italy, over 15,000 health personnel were infected as of mid-April, with 127 physicians succumbing to the virus after treating COVID-19 patients (de Girolamo et al., 2020). Healthcare workers in Italy were infected twice as high as healthcare workers in China, and it is not clear why. An important lesson from Italy was the focus that is needed on healthcare staff working directly with COVID-19 patients (de Girolamo et al., 2020).

\subsection{Home Care Workers}

An important occupational group that is often overlooked are home care workers. These workers are employed in long term homes or participation homes that primarily look after the elderly and adults who require assisted living. Long term care facilities are predominately made up of unregulated healthcare workers such as personal support workers and care aids (Estabrooks, Squires, Carleton, Cummings, \& Norton, 2015). The remaining home care workforce is made up of nursing staff, including registered nurses (RNs) and licensed practical nurses (LPNs) (Squires et al., 2019). Although nurses working in hospital settings have been shown to have a higher risk for health problems compared to nurses in other health settings (Hoben et al., 2017), this is quite different during the pandemic. Given the increase of COVID-19 in long term care facilities home care workers are continuously exposed to patients with COVID-19 and stress related to the severity of COVID-19 in the elderly. In North America, there were many COVID-19 outbreaks reported across long term care facilities making it clear that pandemic planning and infection control were not implemented quickly enough.

A recent study in 2019 reported younger nurses (both RNs and LPNs) to have significantly poorer mental health and burnout-emotional compared to older nurses and may be attributed to older nurses having higher seniority and more control over shift choices (Squires et al., 2019). It was also reported that LPNs were more likely to take on nursing activities that were beyond their scope of practice which often leads to increased burnout (Squires et al., 2019). Another study in 2015 identified that care aid workers reported higher levels of job efficacy, while also reporting higher levels of burnout. These workers were more likely to have been born outside of Canada with English not being their first language (Estabrooks et al., 2015). Given that many of the personal support and care aid workers are unregulated, there is a continued lack of information on the characteristics of these workers (Estabrooks et al., 2015). To our knowledge there are currently no published studies on the mental health issues faced by home care workers during the course of the pandemic. Other concerns among these workers during the pandemic include low pay, extreme staffing shortages, employment at multiple homes increasing risk of infection and infection spread, residents leaving for outside appointments, and a lack of personal protective equipment (Victorian Order of Nurses for Canada (VON Canada), 2020). Shortcomings of healthcare systems, like in Canada, were identified through this pandemic, specifically in that the primary focus are hospitals rather than other facilities like long term care homes (Registered Nurses' Association of Ontario (RNAO), 2020).

\subsection{Other Frontline Workers}

Other essential workers in the frontlines include those employed in protective services often referred to as first responders (ex. police, firefighters), supply of food/medicine such as grocery or retail, critical infrastructure (ex. 
transportation), emergency shelters, supportive housing, social services, correctional institutions, and youth justice facilities. The mental health issues concerning these workers should also be investigated to ultimately protect the mental health of these workers during this pandemic, and for future outbreaks.

\subsection{Recommendations: Mitigating Factors for Mental Health Issues}

Mitigating methods addressed by various governments include increasing the number of healthcare staff to reduce the workload of existing healthcare staff, establishing psychological intervention teams to provide psychological services, and providing services through counseling, psychotherapy, and brochures (Kang et al., 2020; Zhou et al., 2020). Individual mental health targeted interventions are also being used to provide immediate access to support groups, and online or phone counselling. This can ultimately reduce stress and burnout among workers and help to strengthen the healthcare workforce (Bansal et al., 2020; Harrad \& Francesco, 2018).

Self-care is also often recognized as a key mitigating factor in mental health burden influenced by sleep, exercise, and diet (Gold et al., 2020). However, given the dire circumstances of the pandemic, self-care is challenging for frontline workers to achieve (Gold et al., 2020). There are also multiple community efforts towards improving the wellness of frontline workers, such as thanking workers through social media, while many businesses are offering free services for frontline workers (ex. free meals) (Bansal et al., 2020). Some countries have provided financial assistance or increased pay, free child care, and additional family services to ease financial or child care burden (Bansal et al., 2020; Gold et al., 2020). Social media can also be beneficial for frontline workers helping them connect with loved ones and other professionals and gain up to date best practice information (Bansal et al., 2020). Social support is an important mitigating factor, however this can be difficult for healthcare workers who often work long hours, neglect social relationships, or are in fear of possibly infecting others (Blake et al., 2020; Mo et al., 2020; Wu et al., 2020).

There is an important need for system-level changes to reach a larger number of affected workers (Wu et al., 2020). Changes include strict health and safety hospital policies, adequate resources including replenished personal protective equipment (Wu et al., 2020), increased pay, increased food and supplies. When examining home care facilities in particular, the Registered Nurses' Association of Ontario (RNAO) have recently provided a comprehensive framework to guide the healthcare system through COVID-19. The framework provides recommendations for establishing person-centered focus for disease prevention and health promotion, expanding primary care teams, integrating nursing and retirement homes into enhanced community care plans and funding, utilizing evidence-based practice, improving access and person-centered care through digital health technologies (RNAO, 2020). There is also a need for rigorous annual inspections for workplace health and safety across long term care facilities. Disease protocols and coping mechanisms need to be adapted for long term care facilities, with appropriate education and training for workers (Harrad \& Francesco, 2018). The development of a free and accessible digital intervention which encompasses digital learning packages to provide evidence-based support for health care workers may also be valuable (Blake et al., 2020). The digital intervention focuses on normalizing psychological responses during a public health crisis, and promoting help-seeking behaviour and self-care. (Blake et al., 2020). Lastly, there is a need for universal testing protocols for all frontline workers, not just hospital-based workers.

\subsection{Limitations \& Strengths}

There are several limitations that are expected with a scoping review, specifically that there are currently limited studies on the mental health impact among frontline workers during the COVID-19 pandemic. The studies included in this review include very recent data originating mostly from China. Both original articles and letters to the editors were included to provide a wider range of data and to encompass a better understanding of the mental health impact in frontline workers. A majority of included original studies were of cross-sectional design, representative of data captured at only one point in time and not as a longitudinal approach. However, these studies provide findings that are comparable and consistent with one another. Studies included were not assessed for bias, it is expected that bias may exist across included studies. We also aimed to avoid selection bias by identifying and reviewing all available published data on the topic.

This scoping review is valuable as we reviewed a novel topic with very recent literature. The results of this review provide useful insights for mental health awareness and intervention related to the COVID-19 pandemic, an unprecedented time in history. Review of current studies provides better understanding of common mental health themes reported by frontline workers which can lead to better mental health evaluation and intervention. There is opportunity for healthcare systems to plan, mobilize, and develop new resources and interventions to address the major mental health challenges stemming from COVID-19 (Choi et al., 2020). This will require hospitals, healthcare workers, and mental health systems to work together effectively to intervene across different 
occupations. Creating specific programs that are catered towards each unique workplace, with mitigating factors in mind, will provide the necessary resources for frontline workers.

\section{Conclusion}

In conclusion, mental health is important for maintaining a healthy and sustainable frontline workforce, especially across the healthcare system. By understanding the common mental health issues experienced by frontline workers, the mental wellbeing of these workers can be improved and we can be better prepared for future public health crises.

\section{Acknowledgements}

None.

\section{Funding}

This research did not receive any specific grant from funding agencies in the public, commercial, or not-for-profit sectors.

\section{Author Contributions}

All authors (JS, TJ, DV, AS) were involved and contributed to the study conception and design. The literature search and data synthesis were performed by JS and TJ. All authors (JS, TJ, DV, AS) contributed to the interpretation of the data, drafting and critically revising the manuscript. All authors (JS, TJ, DV, AS) have read and approved the final manuscript.

\section{Competing Interests Statement}

The authors declare that they have no conflict of interest.

\section{References}

Abdulah, D. M., \& Musa, D. H. (2020). Insomnia and Stress of Physicians during COVID-19 Outbreak. Sleep Medicine: X, 100017. https://doi.org/10.1016/j.sleepx.2020.100017.

Barello, S., Palamenghi, L., \& Graffigna, G. (2020). Burnout and somatic symptoms among frontline healthcare professionals at the peak of the Italian COVID-19 pandemic. Psychiatry Research, 113-129. https://doi.org/10.1016/j.psychres.2020.113129

Blake, H., Bermingham, F., Johnson, G., \& Tabner, A. (2020). Mitigating the psychological impact of COVID-19 on healthcare workers: a digital learning package. International Journal of Environmental Research and Public Health, 17(9), 2997.

Bansal, P., Bingemann, T. A., Greenhawt, M., Mosnaim, G., Nanda, A., Oppenheimer, J., ... \& Shaker, M. (2020). Clinician wellness during the COVID-19 pandemic: extraordinary times and unusual challenges for the allergist/immunologist. The Journal of Allergy and Clinical Immunology: In Practice. https://doi.org/10.1016/j.jaip.2020.04.001.

Choi, K. R., Heilemann, M. V., Fauer, A., \& Mead, M. (2020). A second pandemic: Mental health spillover from the novel coronavirus (COVID-19). Journal of the American Psychiatric Nurses Association, 1078390320919803.

De Girolamo, G., Cerveri, G., Clerici, M., Monzani, E., Spinogatti, F., Starace, F., ... \& Vita, A. (2020). Mental health in the coronavirus disease 2019 emergency-the Italian response. JAMA psychiatry. https://doi.org/10.1001/jamapsychiatry.2020.1276

Du, J., Dong, L., Wang, T., Yuan, C., Fu, R., Zhang, L., ... \& Bouey, J. (2020). Psychological symptoms among frontline healthcare workers during COVID-19 outbreak in Wuhan. General hospital psychiatry. https://doi.org/10.1016/j.genhosppsych.2020.03.011

Elbay, R. Y., Kurtulmuş, A., Arpacioğlu, S., \& Karadere, E. (2020). Depression, Anxiety, Stress Levels of Physicians and Associated Factors In Covid-19 Pandemics. Psychiatry Research, 113130. https://doi.org/10.1016/j.psychres.2020.113130

Estabrooks, C. A., Squires, J. E., Carelton, H. L., Cummings, G. G., \& Norton, P. G. (2015). Who is looking after mom and dad? Unregulated workers in Canadian long-term care homes. Canadian Journal of Aging, 34(1), 47- 59.

Gold, J. A. (2020). Covid-19: adverse mental health outcomes for healthcare workers. BMJ, 369, m1815. https://doi.org/10.1136/bmj.m1815 
Government of Canada. (2020). Guidance on essential services and functions in Canada during the COVID-19 pandemic. Public Safety Canada. Retrieved on March 15, 2020 from scrt/crtcl-nfrstrctr/esf-sfe-en.aspx

Rachel, H., \& Francesco, S. (2018). Factors associated with and impact of burnout in nursing and residential home care workers for the elderly. Acta Bio Medica: Atenei Parmensis, 89(Suppl 7), 60-69.

Hoben, M., Knopp-Sihota, J. A., Nesari, M., Chamberlain, S. A., Squires, J. E., Norton, P. G., ... \& Estabrooks, C. A. (2017). Health of health care workers in Canadian nursing homes and pediatric hospitals: a cross-sectional study. CMAJ open, 5(4), E791.

Huang, Y., \& Zhao, N. (2020). Mental health burden for the public affected by the COVID-19 outbreak in China: Who will be the high-risk group? Psychology, Health \& Medicine, 1-12. https://doi.org/10.1080/13548506.2020.1754438

Kang, L., Ma, S., Chen, M., Yang, J., Wang, Y., Li, R., ... \& Hu, S. (2020). Impact on mental health and perceptions of psychological care among medical and nursing staff in Wuhan during the 2019 novel coronavirus disease outbreak: A cross-sectional study. Brain, behavior, and immunity. https://doi.org/10.1016/j.bbi.2020.03.028

Lai, J., Ma, S., Wang, Y., Cai, Z., Hu, J., Wei, N., ... \& Tan, H. (2020). Factors associated with mental health outcomes among health care workers exposed to coronavirus disease 2019. JAMA network open, 3(3), e203976-e203976. https://doi.org/10.1001/jamanetworkopen.2020.3976

Levac, D., Colquhoun, H., \& O’Brien, K. K. (2010). Scoping studies: advancing the methodology. Implementation Science, 5(69).

Li, X., Yu, H., Bian, G., Hu, Z., Liu, X., Zhou, Q., ... \& Zhou, D. (2020). Prevalence, risk factors, and clinical correlates of insomnia in volunteer and at home medical staff during the COVID-19. Brain, Behavior, and Immunity. https://doi.org/10.1016/j.bbi.2020.05.008

Liu, C. Y., Yang, Y., Xhang, X. M., Xu, X., Dou, Q., Zhang, W., ... \& Cheng, A. S. K. (2020). The prevalence and influencing factors in anxiety in medical workers fighting COVID-19 in China: a cross-sectional survey. Epidemiology and Infection, 148, e98, 1-7. https://doi.org/10.1017/S0950268820001107

Lu, W., Wang, H., Lin, Y., \& Li, L. (2020). Psychological status of medical workforce during the COVID-19 pandemic: a cross-sectional study. Psychiatry Research, 288. https://doi.org/10.1016/j.psychres.2020.112936

Min Lee, S., Kang, W. S., Cho, A., Kim, T., \& Park, J. K. (2018). Psychological impact of the 2015 MERS outbreak on hospital workers and quarantined hemodialysis patients. Comprehensive Psychiatry, 87, 123-127.

Mo, Y., Deng, L., Zhang, L., Lang, Q., Liao, C., Wang, N., ... \& Huang, H. (2020). Work stress among Chinese nurses to support Wuhan in fighting against COVID-19 epidemic. Journal of nursing management. https://doi.org/10.1111/jonm.13014

Neto, M. L. R., Almeida, H. G., Esmeraldo, J. D. A., Nobre, C. B., Pinheiro, W. R., de Oliveira, C. R. T., ... \& Lima, C. K. T. (2020). When health professionals look death in the eye: the mental health of professionals who deal daily with the 2019 coronavirus outbreak. Psychiatry Research, 112972. https://doi.org/10.1016/j.psychres.2020.112972.

Park, J., Lee, E., Park, N., \& Choi, Y. H. (2018). Mental health of nurses working at a government-designated hospital during a MERS-CoV outbreak: a cross-sectional study. Archives of Psychiatric Nursing, 32, 2-6.

Qi, J., Xu, J., Li, B. Z., Huang, J. S., Yang, Y., Zhang, Z. T., ... \& Ni, X. H. (2020). The evaluation of sleep disturbances for Chinese frontline medical workers under the outbreak of COVID-19. Sleep Medicine. https://doi.org/10.1016/j.sleep.2020.05.023

Registered Nurses' Association of Ontario (RNAO). (2020). Pandemic puts health system to the test: nurses have answers for shortfalls. RNAO. Retrieved from https://rnao.ca/news/media-releases/2020/05/12/pandemicputs-health-system-test-nurses-have-answers-shortfalls

Squires, J. E., Baumbusch, J., Varin, M. D., MacDonald, I., Chamberlain, S., Boström, A. M., ... \& Estabrooks, C. A. (2019). A profile of regulated nurses employed in Canadian long-term care facilities. Canadian Journal on Aging/La Revue canadienne du vieillissement, 38(2), 130-142. https://doi.org/10.1017/S0714980818000478

Sritharan, J., \& Sritharan, A. (2020). Emerging mental health issues from the novel Coronavirus (COVID-19) pandemic. Journal of Health and Medical Sciences, 3(2).

Sun, N., Wei, L., Shi, S., Jiao, D., Song, R., Ma, L., ... \& Liu, S. (2020). A qualitative study on the psychological 
experience of caregivers of COVID-19 patients. American journal of infection control, 48(6), 592-598. https://doi.org/10.1016/j.ajic.2020.03.018

Victorian Order of Nurses for Canada (VON Canada). (2020). Ontario's home care sector needs help now - a note from VON CEO, Jo-Anne Poirier. Retrieved on May 26, 2020 from home-care-sector-needs-help-now-note-von-ceo-jo-anne-poirier

Wang, S., Xie, L., Xu, Y., Yu, S., Yao, B., \& Xiang, D. (2020). Sleep disturbances among medical workers during the outbreak of COVID-2019. Occupational Medicine (Oxford, England). https://doi.org/10.1093/occmed/kqaa074

Wu, P. E., Styra, R., \& Gold, W. L. (2020). Mitigating the psychological effects of COVID-19 on health care workers. CMAJ, 192(17), E459-E460. https://doi.org/10.1503/cmaj.200519

Xiao, H., Zhang, Y., Kong, D., Li, S., \& Yang, N. (2020a). The effects of social support on sleep quality of medical staff treating patients with coronavirus disease 2019 (COVID-19) in January and February 2020 in China. Medical science monitor: international medical journal of experimental and clinical research, 26, e923549-1.

Xiao, X., Zhu, X., Fu, S., Hu, Y., Li, X., \& Xiao, J. (2020b). Psychological impact of healthcare workers in China during COVID-19 pneumonia epidemic: a multi-center cross-sectional survey investigation. Journal of Affective Disorders. https://doi.org/10.1016/j.jad.2020.05.081

Xu, J., Xu, Q. H., Wang, C. M., \& Wang, J. (2020). Psychological status of surgical staff during the COVID-19 outbreak. Psychiatry research, 112955. https://doi.org/10.1016/j.psychres.2020.112955

Zhang, C., Yang, L., Liu, S., Ma, S., Wang, Y., Cai, Z., ... \& Zhang, B. (2020a). Survey of insomnia and related social psychological factors among medical staff involved in the 2019 novel coronavirus disease outbreak. Frontiers in Psychiatry, 11, 306. https://doi.org/10.3389/fpsyt.2020.00306

Zhang, S. X., Liu, J., Jahanshahi, A. A., Nawaser, K., Yousefi, A., Li, J., ... \& Sun, S. (2020b). At the height of the storm: healthcare staff's health conditions and job satisfaction and their associated predictors during the epidemic peak of COVID-19. Brain, Behavior, and Immunity. https://doi.org/10.1016/j.bbi.2020.05.010

Zhou, Y., Zhou, Y., Song, Y., Ren, L., Ng, C. H., Xiang, Y., ... \& Tang, Y. (2020). Tackling the mental health burden of frontline healthcare staff in the COVID-19 pandemic: China's experiences. Psychological Medicine, 1-5. https://doi.org/10.1017/S0033291720001622 
Supplementary Table 1. List of search strategies used to identify all included studies in the scoping review, 2020.

\begin{tabular}{|c|c|c|c|c|c|}
\hline Search engine & $\begin{array}{l}\text { Date of } \\
\text { Search }\end{array}$ & Search Words & $\begin{array}{l}\text { Number of } \\
\text { studies } \\
\text { Retrieved }\end{array}$ & $\begin{array}{l}\text { Number of } \\
\text { studies } \\
\text { excluded* }\end{array}$ & $\begin{array}{c}\text { Number of } \\
\text { Unique } \\
\text { Studies } \\
\text { Reviewed }\end{array}$ \\
\hline PubMed & 4-May-20 & $\begin{array}{l}\text { (((mental health[Title/Abstract]) AND frontline[Title/Abstract]) AND coronavirus[Title/Abstract]) OR } \\
\text { COVID-19 [Title/Abstract] })\end{array}$ & 6 & 1 & 5 \\
\hline PubMed & 4-May-20 & (((mental health[Title/Abstract]) AND front line [Title/Abstract]) AND COVID-19[Title/Abstract]) & 4 & 4 & 0 \\
\hline PubMed & 4-May-20 & $\begin{array}{llll}(((\text { psychological distress[Title/Abstract] }) & \text { AND } & \text { frontline[Title/Abstract] }) & \text { AND } \\
\text { coronavirus[Title/Abstract] }) & & \end{array}$ & 2 & 2 & 0 \\
\hline PubMed & 4-May-20 & $\begin{array}{l}(((\text { mental health[Title/Abstract] }) \text { AND nurse[Title/Abstract] }) \text { AND coronavirus[Title/Abstract]) OR } \\
\text { COVID-19 [Title/Abstract] })\end{array}$ & 5 & 0 & 5 \\
\hline PubMed & 4-May-20 & ((mental health[Title/Abstract]) AND nurses[Title/Abstract]) AND coronavirus[Title/Abstract] & 7 & 5 & 2 \\
\hline PubMed & 4-May-20 & (((mental health[Title/Abstract]) AND physician[Title/Abstract]) AND coronavirus[Title/Abstract]) & 0 & 0 & 0 \\
\hline PubMed & 4-May-20 & $((($ mental health[Title/Abstract]) AND medical staff[Title/Abstract]) AND coronavirus[Title/Abstract]) & 5 & 1 & 4 \\
\hline PubMed & 4-May-20 & $\begin{array}{l}(((\text { mental health[Title/Abstract] }) \\
\text { coronavirus[Title/Abstract])) })\end{array}$ & 2 & 1 & 1 \\
\hline PubMed & 4-May-20 & $\begin{array}{l}(((\text { mental health[Title/Abstract] }) \\
\text { coronavirus[Title/Abstract] })\end{array}$ & 0 & 0 & 0 \\
\hline PubMed & 4-May-20 & $\begin{array}{lllll}(((\text { mental health[Title/Abstract] }) & \text { AND } & \text { first } & \text { responder[Title/Abstract] }) & \text { AND } \\
\text { coronavirus[Title/Abstract] }))) & & & & \end{array}$ & 0 & 0 & 0 \\
\hline PubMed & 4-May-20 & $((($ mental health[Title/Abstract]) AND firefighter[Title/Abstract]) AND coronavirus[Title/Abstract]))) & 0 & 0 & 0 \\
\hline PubMed & 4-May-20 & $((($ mental health[Title/Abstract]) AND police[Title/Abstract]) AND coronavirus[Title/Abstract]))) & 0 & 0 & 0 \\
\hline PubMed & 4-May-20 & $\begin{array}{l}(((\text { mental health[Title/Abstract] }) \\
\text { coronavirus[Title/Abstract] })))\end{array}$ & 0 & 0 & 0 \\
\hline PubMed & 4-May-20 & $((($ mental health[Title/Abstract]) AND occupation[Title/Abstract]) AND coronavirus[Title/Abstract]))) & 1 & 1 & 0 \\
\hline APA PsycInfo & 4-May-20 & $\mathrm{ab}$ (mental health) AND ab(frontline worker) AND ab(coronavirus) found 0 results. & 0 & 0 & 0 \\
\hline APA PsycInfo & 4-May-20 & $\mathrm{ab}$ (mental health) AND ab(frontline) AND ab(coronavirus) found 0 results. & 0 & 0 & 0 \\
\hline APA PsycInfo & 4-May-20 & $a b$ (mental health) AND ab(nurse) AND ab(coronavirus) & 1 & 1 & 0 \\
\hline APA PsycInfo & 4-May-20 & ab(mental health) AND ab(medical staff) AND ab(coronavirus) & 1 & 1 & 0 \\
\hline
\end{tabular}




\begin{tabular}{|c|c|c|c|c|c|}
\hline APA PsycInfo & 4-May-20 & $a b$ (mental health) AND ab(hospital staff) AND ab(coronavirus) & 0 & 0 & 0 \\
\hline APA PsycInfo & 4-May-20 & $\mathrm{ab}$ (mental health) AND ab(physician) AND ab(coronavirus) & 0 & 0 & 0 \\
\hline APA PsycInfo & 4-May-20 & $\mathrm{ab}$ (mental health) AND ab(personal support worker) AND ab(coronavirus) & 0 & 0 & 0 \\
\hline APA PsycInfo & 4-May-20 & $\mathrm{ab}$ (mental health) AND ab(first responder) AND ab(coronavirus) & 0 & 0 & 0 \\
\hline APA PsycInfo & 4-May-20 & $\mathrm{ab}$ (mental health) AND ab(protective services) AND ab(coronavirus) & 0 & 0 & 0 \\
\hline APA PsycInfo & 4-May-20 & $\mathrm{ab}$ (mental health) AND ab(police) AND ab(coronavirus) & 0 & 0 & 0 \\
\hline APA PsycInfo & 4-May-20 & $\mathrm{ab}$ (mental health) AND ab(firefighter) AND ab(coronavirus) & 0 & 0 & 0 \\
\hline APA PsycInfo & 4-May-20 & $\mathrm{ab}$ (mental health) AND ab(occupation) AND ab(coronavirus) & 2 & 1 & 1 \\
\hline CINAHL Plus & 4-May-20 & $\mathrm{AB}$ mental health $\mathrm{AND} \mathrm{AB}$ front line $\mathrm{AND} \mathrm{AB}$ coronavirus & 1 & 0 & 1 \\
\hline CINAHL Plus & 4-May-20 & $\mathrm{AB}$ mental health $\mathrm{AND} \mathrm{AB}$ frontline $\mathrm{AND} \mathrm{AB}$ coronavirus & 2 & 2 & 0 \\
\hline CINAHL Plus & 4-May-20 & $\mathrm{AB}$ mental health $\mathrm{AND} \mathrm{AB}$ nurse $\mathrm{AND} \mathrm{AB}$ coronavirus & 2 & 2 & 0 \\
\hline CINAHL Plus & 4-May-20 & $\mathrm{AB}$ mental health $\mathrm{AND} \mathrm{AB}$ nurses $\mathrm{AND} \mathrm{AB}$ coronavirus & 2 & 2 & 0 \\
\hline CINAHL Plus & 4-May-20 & AB mental health AND AB medical staff AND AB coronavirus & 0 & 0 & 0 \\
\hline CINAHL Plus & 4-May-20 & $\mathrm{AB}$ mental health $\mathrm{AND} \mathrm{AB}$ hospital staff AND AB coronavirus & 0 & 0 & 0 \\
\hline CINAHL Plus & 4-May-20 & $\mathrm{AB}$ mental health $\mathrm{AND} A \mathrm{AB}$ physician $\mathrm{AND} \mathrm{AB}$ coronavirus & 1 & 1 & 0 \\
\hline CINAHL Plus & 4-May-20 & $\mathrm{AB}$ mental health $\mathrm{AND}$ AB personal support worker $\mathrm{AND}$ AB coronavirus & 0 & 0 & 0 \\
\hline CINAHL Plus & 4-May-20 & $\begin{array}{l}\mathrm{AB} \text { mental health } \mathrm{AND} A \mathrm{AB} \text { first responders or firefighters or paramedics or police or emergency } \\
\text { services or ems AND } \mathrm{AB} \text { coronavirus }\end{array}$ & 0 & 0 & 0 \\
\hline CINAHL Plus & 4-May-20 & $\mathrm{AB}$ mental health $\mathrm{AND} A \mathrm{AB}$ protective services $\mathrm{AND} \mathrm{AB}$ coronavirus & 0 & 0 & 0 \\
\hline CINAHL Plus & 4-May-20 & $\mathrm{AB}$ mental health $\mathrm{AND} \mathrm{AB}$ police $\mathrm{AND} \mathrm{AB}$ coronavirus & 0 & 0 & 0 \\
\hline CINAHL Plus & 4-May-20 & $\mathrm{AB}$ mental health $\mathrm{AND} \mathrm{AB}$ firefighter $\mathrm{AND} \mathrm{AB}$ coronavirus & 0 & 0 & 0 \\
\hline CINAHL Plus & 4-May-20 & AB mental health AND AB occupation AND AB coronavirus & 0 & 0 & 0 \\
\hline Pubmed & 14-Мay-20 & $\begin{array}{l}(((()(()(()((()(()(()(()(((\text { healthcare) OR healthcare worker) OR physician) OR primary care) OR } \\
\text { doctor) OR nurse) OR doctors) OR nurses) OR frontline) OR front line worker) OR primary care } \\
\text { physician) OR hospital staff) OR medical system) OR healthcare staff) OR cleaners) OR front-line } \\
\text { staff) OR frontline staff) OR paramedic) OR paramedics) OR health specialist) OR respiratory } \\
\text { therapist) OR therapist) OR firefighter) OR police) OR first responder })) \text { AND }(((((C O V I D-19) \text { OR } \\
\text { covid-19) OR coronavirus) OR "severe acute respiratory syndrome coronavirus 2") OR } \\
\text { "SARS-CoV-2")) AND ("2020/01/01"[PDat] : "2020/12/31"[PDat] ))) AND mental health }\end{array}$ & 148 & 118 & 30 \\
\hline
\end{tabular}




\begin{tabular}{|c|c|c|c|c|c|}
\hline APA PsycInfo & 28-May-20 & $\begin{array}{l}\text { healthcare OR (healthcare worker) OR physician OR doctor OR nurse OR (hospital staff) OR (medical } \\
\text { staff) AND COVID-19 OR coronavirus AND (mental health) [restricted to publications past may 14, } \\
\text { 2020] }\end{array}$ & 170 & 170 & 0 \\
\hline Pubmed & 29-May-20 & $\begin{array}{l}((((()((()((()(()((()(()(((\text { healthcare) OR healthcare worker) OR physician) OR primary care) OR } \\
\text { doctor) OR nurse) OR doctors) OR nurses) OR frontline) OR front line worker) OR primary care } \\
\text { physician) OR hospital staff) OR medical system) OR healthcare staff) OR cleaners) OR front-line } \\
\text { staff) OR frontline staff) OR paramedic) OR paramedics) OR health specialist) OR respiratory } \\
\text { therapist) OR therapist) OR firefighter) OR police) OR first responder })) \text { AND }((((\text { COVID-19) OR } \\
\text { covid-19) OR coronavirus) OR "severe acute respiratory syndrome coronavirus 2") OR } \\
\text { "SARS-CoV-2")) AND ( "2020/01/01"[PDat] : "2020/12/31"[PDat] ))) AND mental health }\end{array}$ & 328 & 322 & 6 \\
\hline TOTAL & & & 684 & 634 & 50 \\
\hline
\end{tabular}

*Studies excluded if they did not meet the inclusion criteria or were duplicates of already included studies.

\section{Copyrights}

Copyright for this article is retained by the author(s), with first publication rights granted to the journal.

This is an open-access article distributed under the terms and conditions of the Creative Commons Attribution license (http://creativecommons.org/licenses/by/4.0/). 